\title{
Notes on entropic characteristics of quantum channels
}

\author{
Alexey E. Rastegin \\ Department of Theoretical Physics, Irkutsk State University, Gagarin Bv. 20, Irkutsk 664003, Russia
}

\begin{abstract}
One of most important issues in quantum information theory concerns transmission of information through noisy quantum channels. We discuss few channel characteristics expressed by means of generalized entropies. Such characteristics can often be dealt in line with more usual treatment based on the von Neumann entropies. For any channel, we show that the $q$-average output entropy of degree $q \geq 1$ is bounded from above by the $q$-entropy of the input density matrix. Concavity properties of the $(q, s)$-entropy exchange are considered. Fano type quantum bounds on the $(q, s)$ entropy exchange are derived. We also give upper bounds on the map $(q, s)$-entropies in terms of the output entropy, corresponding to the completely mixed input.
\end{abstract}

PACS numbers: 03.67.-a, 03.67.Hk, 02.10.Ud

\section{INTRODUCTION}

The quantum information theory treats quantum states and effects as tools for information processing [1, 2]. Many of the important characteristics of quantum channels and noise are expressed in terms of entropic measures. The Shannon entropy of a classical probability distribution and the von Neumann entropy of a density matrix are both of great importance. In addition, some extensions of these entropic functionals were found to be fruitful. Most important of them are the Rényi [3] and Tsallis entropies [4], the quantum versions of which are frequently used as well. A common treatment of these and some other entropies in terms of unified $(q, s)$-entropies was given in Ref. [5]. For many values of parameters $q$ and $s$, the quantum unified $(q, s)$-entropy enjoys properties similarly to the standard von Neumann entropy [6]. Entropic functionals have found a broad use in studying features of quantum channels [1].

In information-theoretical context, various applications of quantum entropies are considered in Ref. [2]. The entropy exchange [7] and the map entropy [8] are widely known among different entropic characteristics. In Ref. [9], the uncertainty relations for a single quantum operation are expressed in terms of the map and receiver entropies. Entropic uncertainty relations are still the subject of active research (see the reviews [10, 11] and references therein). Additivity properties of map entropies with respect to the tensor product of two channels are the subject of active research [12]. For the minimum output entropy, this question is regarded to be even more important [13, 14]. Together with the von Neumann entropy, the quantum Rényi entropy has been applied for these aims. In Ref. [15], some properties of quantum channels have been considered with use of unified entropies. In particular, the known inequality of Lindblad [16] with the entropy exchange was extended to the case of unified entropies.

In this paper, we further study channel characteristics based on generalized quantum entropies. The paper is organized as the following: The preliminary material is reviewed in Section III In Section III we derive an upper bound on the $q$-average output entropy of degree $q \geq 1$ in terms of the corresponding entropy of the input. This bound, which holds for arbitrary quantum channel, is explicitly shown with the depolarizing channel. In Section IV] concavity properties of the $(q, s)$-entropy exchange are examined. Fano type quantum inequalities for the $(q, s)$ entropy exchange are obtained in Section $\nabla$ These inequalities cover all the acceptable values of the parameters $q$ and $s$. Upper bounds on the map $(q, s)$-entropy in terms of the output entropy of completely mixed input are presented in Section [VI] In Section [II], we conclude the paper.

\section{DEFINITION AND NOTATION}

In this section, the preliminary material is given. Let $\mathcal{L}(\mathcal{H})$ be the space of linear operators on $d$-dimensional Hilbert space $\mathcal{H}$. By $\mathcal{L}_{+}(\mathcal{H})$ and $\mathcal{L}_{++}(\mathcal{H})$, we respectively denote the set of positive semidefinite operators and the set of strictly positive ones. A density operator $\boldsymbol{\rho} \in \mathcal{L}_{+}(\mathcal{H})$ has unit trace, i.e., $\operatorname{Tr}(\boldsymbol{\rho})=1$. For probability distribution $\left\{p_{j}\right\}$, the non-extensive entropy of degree $q$ is defined as [4]

$$
H_{q}\left(p_{j}\right):=\frac{1}{1-q}\left(\sum_{j} p_{j}^{q}-1\right)=-\sum_{j} p_{j}^{q} \ln _{q}\left(p_{j}\right) .
$$

Here the $q$-logarithm of argument $x>0$ is put for $q>0 \neq 1$ as $\ln _{q}(x)=\left(x^{1-q}-1\right) /(1-q)$. In non-extensive statistical mechanics, the entropy (11) is commonly referred to as Tsallis $q$-entropy. Up to a factor, the right-hand side of Eq. (11) was also put within information theoretic context in Ref. [17]. For any $p \in[0 ; 1]$, the binary Tsallis entropy is

$$
\widetilde{H}_{q}(p)=-p^{q} \ln _{q}(p)-(1-p)^{q} \ln _{q}(1-p) .
$$


For physical quantity $A$, which takes the value $a_{j}$ with probability $p_{j}$, the unnormalized $q$-average is defined as [18]

$$
\langle A\rangle_{q}:=\sum_{j} p_{j}^{q} a_{j}
$$

Note that the formula (1) can be rewritten as $H_{q}\left(p_{j}\right)=\left\langle-\ln _{q}\left(p_{j}\right)\right\rangle_{q}$. The Rényi entropies form another important class of generalized entropic functions. For $q>0 \neq 1$, the Rényi $q$-entropy is defined as [3]

$$
R_{q}\left(p_{j}\right)=\frac{1}{1-q} \ln \left(\sum_{j} p_{j}^{q}\right) .
$$

When $q \rightarrow 1$, the entropies (11) and (4) both recover the Shannon entropy $H_{1}\left(p_{j}\right)=-\sum_{j} p_{j} \ln p_{j}$. In quantum theory, the above entropies have found use in studying measurement uncertainties [9 11, 19 24]. Applications of the entropy (11) in analyzing multiparticle production processes are discussed in Ref. [25].

For density matrix $\boldsymbol{\rho}$, its von Neumann entropy is defined as $\mathrm{H}_{1}(\boldsymbol{\rho})=-\operatorname{Tr}(\boldsymbol{\rho} \ln \boldsymbol{\rho})$. This is a quantum counterpart of the Shannon entropy. Extensions of the von Neumann entropy are obtained from Eqs. (11) and (4) by replacing the sums with the corresponding traces. The quantum $q$-entropy of density operator $\rho$ is written as [26]

$$
\mathrm{H}_{q}(\boldsymbol{\rho}):=\frac{\operatorname{Tr}\left(\boldsymbol{\rho}^{q}\right)-1}{1-q} .
$$

In Ref. [5], $\mathrm{Hu}$ and Ye proposed the class of more general extensions of the standard entropic function. For positive $q \neq 1$ and any $s \neq 0$, the unified $(q, s)$-entropy of probability distribution $\left\{p_{j}\right\}$ is defined by

$$
E_{q}^{(s)}\left(p_{j}\right):=\frac{1}{(1-q) s}\left[\left(\sum_{j} p_{j}^{q}\right)^{s}-1\right]
$$

The quantum unified $(q, s)$-entropy of density operator $\boldsymbol{\rho}$ is defined as

$$
\mathrm{E}_{q}^{(s)}(\boldsymbol{\rho}):=\frac{1}{(1-q) s}\left\{\left[\operatorname{Tr}\left(\boldsymbol{\rho}^{q}\right)\right]^{s}-1\right\}
$$

for $q>0 \neq 1$ and any $s \neq 0[5]$. In the limit $s \rightarrow 0$, the right-hand side of Eq. (7]) leads to the quantum Rényi entropy

$$
\mathrm{R}_{q}(\boldsymbol{\rho}):=\frac{1}{1-q} \ln \left[\operatorname{Tr}\left(\boldsymbol{\rho}^{q}\right)\right] .
$$

In the case $q=1$, we obtain the von Neumann entropy $-\operatorname{Tr}(\boldsymbol{\rho} \ln \boldsymbol{\rho})$. For the completely mixed state $\boldsymbol{\rho}_{*}=\mathbb{1} / d$, the entropy (7) reaches its maximum [5]

$$
\mathrm{E}_{q}^{(s)}\left(\boldsymbol{\rho}_{*}\right)=\frac{1}{s} \ln _{q}\left(d^{s}\right)
$$

For the Tsallis and Renyi entropies, we then have $\mathrm{H}_{q}\left(\boldsymbol{\rho}_{*}\right)=\ln _{q}(d)$ and $\mathrm{R}_{q}\left(\boldsymbol{\rho}_{*}\right)=\ln d$, respectively.

Consider a linear map $\Phi$ that takes elements of $\mathcal{L}(\mathcal{H})$ to elements of $\mathcal{L}\left(\mathcal{H}^{\prime}\right)$, and also satisfies the condition of complete positivity. Let id ${ }^{R}$ be the identity map on $\mathcal{L}\left(\mathcal{H}_{R}\right)$, where the space $\mathcal{H}_{R}$ is assigned to auxiliary reference system. The complete positivity implies that $\Phi \otimes \mathrm{id}^{R}$ transforms any positive operator into a positive operator again for each dimension of the extended space. Such linear maps are typically called quantum operations [1] or super-operators [27]. Each completely positive map can be written in the operator-sum representation, namely

$$
\Phi(\mathrm{X})=\sum_{j} \mathrm{~K}_{j} \mathrm{XK}_{j}^{\dagger}
$$

for all $\mathrm{X} \in \mathcal{L}(\mathcal{H})$. Here the Kraus operators $\mathrm{K}_{j}$ map the input space $\mathcal{H}$ to the output space $\mathcal{H}^{\prime}$ [1, 2]. If the map is trace-preserving, then the Kraus operators satisfy the condition

$$
\sum_{j} \mathrm{~K}_{j}^{\dagger} \mathrm{K}_{j}=\mathbb{1}
$$

where $\mathbb{1}$ is the identity in $\mathcal{H}$. Trace-preserving quantum operations are often called quantum channels [1]. Let $\boldsymbol{\rho}$ be the input of quantum channel $\Phi$. The $j$ th effect occurs with the probability

$$
p_{j}=\operatorname{Tr}\left(\mathrm{K}_{j}^{\dagger} \mathrm{K}_{j} \boldsymbol{\rho}\right)
$$


and leads to the particular (normalized) output

$$
\boldsymbol{\rho}_{j}^{\prime}:=p_{j}^{-1} \mathrm{~K}_{j} \boldsymbol{\rho} \mathrm{K}_{j}^{\dagger} .
$$

Since an informational content of quantum states is measured by means of one or another entropies, various relations between input and output entropies are of great importance. In the following, inequalities of such a kind are formulated in terms of Tsallis $q$-entropies and unified $(q, s)$-entropies.

The Jamiołkowski-Choi representation [28, 29] is another convenient description for completely positive maps. Let $\mathcal{H}_{Q}=\mathcal{H}_{R}=\mathcal{H}$ and $\{|\nu\rangle\}$ be an orthonormal basis in $\mathcal{H}$. To this basis we assign the normalized pure state

$$
\left|\phi_{+}\right\rangle:=\frac{1}{\sqrt{d}} \sum_{\nu=1}^{d}|\nu\rangle \otimes|\nu\rangle,
$$

where $d=\operatorname{dim}(\mathcal{H})$. One introduces the operator $\sigma(\Phi):=\Phi \otimes \operatorname{id}\left(\left|\phi_{+}\right\rangle\left\langle\phi_{+}\right|\right)$, acting on the space $\mathcal{H}^{\otimes 2}$. The matrix $\mathrm{D}(\Phi)=d \boldsymbol{\sigma}(\Phi)$ is usually called dynamical matrix or Choi matrix [8]. For each $\mathbf{X} \in \mathcal{L}(\mathcal{H})$, the action of the map $\Phi$ can be recovered from $\mathrm{D}(\Phi)$ by means of the relation [27]

$$
\Phi(\mathrm{X})=\operatorname{Tr}_{R}\left(\mathrm{D}(\Phi)\left(\mathbb{1} \otimes \mathrm{X}^{T}\right)\right)
$$

where $\mathrm{X}^{T}$ denotes the transpose operator to $\mathrm{X}$. The map $\Phi$ is completely positive, if and only if the matrix $\mathrm{D}(\Phi)$ is positive. The condition $\operatorname{Tr}_{Q}(\mathrm{D}(\Phi))=\mathbb{1}$ is equivalent to that the map $\Phi$ is trace-preserving and the rescaled matrix $\boldsymbol{\sigma}(\Phi)$ is of unit trace. Writing positive semidefinite $\mathrm{D}(\Phi)$ as a sum of one-rank matrices from $\mathcal{L}_{+}\left(\mathcal{H}^{\otimes 2}\right)$, we can get a set of Kraus operators (for details, see Ref. [30] or section 5.2 in Ref. [27]). In the following, the (q, s)-entropy of a quantum channel is defined in terms of its rescaled dynamical matrix.

\section{AN UPPER BOUND ON THE $q$-AVERAGE OUTPUT ENTROPY}

In this section, we will prove an upper bound on the $q$-average of quantum entropies of particular outputs (13). In accordance with Eq. (3), we define the $q$-average entropy as the sum

$$
\left\langle\mathrm{H}_{q}\left(\boldsymbol{\rho}_{j}^{\prime}\right)\right\rangle_{q}:=\sum_{j} p_{j}^{q} \mathrm{H}_{q}\left(\boldsymbol{\rho}_{j}^{\prime}\right) .
$$

This quantity is bounded from above as follows.

Proposition 1 For $q \geq 1$, the q-average output entropy of arbitrary quantum channel is bounded from above as

$$
\left\langle\mathrm{H}_{q}\left(\boldsymbol{\rho}_{j}^{\prime}\right)\right\rangle_{q} \leq \mathrm{H}_{q}(\boldsymbol{\rho}) .
$$

Proof. We take imaginary systems $R$ and $S$, both identical to the original system $Q$. Consider an isometry $\widetilde{\mathrm{V}}: \mathcal{H}_{Q} \rightarrow \mathcal{H}_{Q}^{\prime} \otimes \mathcal{H}_{R} \otimes \mathcal{H}_{S}$, which is defined as

$$
\widetilde{\mathrm{V}}|\psi\rangle=\sum_{j} \mathrm{~K}_{j}|\psi\rangle \otimes\left|\nu_{j}\right\rangle \otimes\left|\nu_{j}\right\rangle
$$

Here $\left\{\left|\nu_{j}\right\rangle\right\}$ is chosen orthonormal basis in $\mathcal{H}_{Q}$. To given input $\boldsymbol{\rho}$ of the channel, we assign the density operator

$$
\omega^{Q^{\prime} R S}:=\widetilde{\mathrm{V}} \boldsymbol{\rho} \widetilde{\mathrm{V}}^{\dagger}=\sum_{i j} \mathrm{~K}_{i} \boldsymbol{\rho} \mathrm{K}_{j}^{\dagger} \otimes\left|\nu_{i}\right\rangle\left\langle\nu_{j}|\otimes| \nu_{i}\right\rangle\left\langle\nu_{j}\right| .
$$

Following Ref. [31], we put the notation $\mathrm{A}_{i j}:=\mathrm{K}_{i} \boldsymbol{\rho} \mathrm{K}_{j}^{\dagger}$, so that $p_{j}=\operatorname{Tr}\left(\mathrm{A}_{j j}\right)$ and $\boldsymbol{\rho}_{j}^{\prime}=p_{j}^{-1} \mathrm{~A}_{j j}$. The corresponding reduced densities are written as

$$
\begin{gathered}
\boldsymbol{\omega}^{Q^{\prime} S}=\sum_{j} \mathrm{~A}_{j j} \otimes\left|\nu_{j}\right\rangle\left\langle\nu_{j}\right|, \\
\boldsymbol{\omega}^{R}=\sum_{j} \operatorname{Tr}\left(\mathrm{A}_{j j}\right)\left|\nu_{j}\right\rangle\left\langle\nu_{j}\right| .
\end{gathered}
$$

With respect to the chosen basis, the matrices $\boldsymbol{\omega}^{Q^{\prime} S}$ and $\boldsymbol{\omega}^{R}=\operatorname{diag}\left[p_{j}\right]$ have a diagonal form. Let $\lambda_{k}^{(j)}$ denote eigenvalues of the positive operator $\mathrm{A}_{j j}$; then

$$
p_{j}=\sum_{k} \lambda_{k}^{(j)},
$$


and the spectrum of $\boldsymbol{\rho}_{j}^{\prime}$ contains $p_{j}^{-1} \lambda_{k}^{(j)}$. The $q$-entropies of the densities (20) and (21) are respectively expressed as

$$
\mathrm{H}_{q}\left(\boldsymbol{\omega}^{Q^{\prime} S}\right)=H_{q}\left(\lambda_{k}^{(j)}\right), \quad \mathrm{H}_{q}\left(\boldsymbol{\omega}^{R}\right)=H_{q}\left(p_{j}\right) .
$$

Due to the definition (1) and the relations (23), the quantity (16) is then rewritten as

$$
\begin{aligned}
\sum_{j} p_{j}^{q} \frac{1}{1-q}\left[\sum_{k}\left(\frac{\lambda_{k}^{(j)}}{p_{j}}\right)^{q}-1\right] & =\frac{1}{1-q} \sum_{j k}\left(\lambda_{k}^{(j)}\right)^{q}-\frac{1}{1-q} \sum_{j} p_{j}^{q} \\
& =\mathrm{H}_{q}\left(\boldsymbol{\omega}^{Q^{\prime} S}\right)-\mathrm{H}_{q}\left(\boldsymbol{\omega}^{R}\right) \leq \mathrm{H}_{q}\left(\boldsymbol{\omega}^{Q^{\prime} R S}\right) .
\end{aligned}
$$

The last step is the triangle inequality for the quantum $q$-entropy of degree $q \geq 1$ [32]. It directly follows from the subadditivity property, which has been conjectured in Ref. [26] and later proved in Ref. [33]. Since the transformation $\widetilde{V}$ is an isometry, the three-partite state (19) and the input state $\rho$ have the same non-zero eigenvalues 31]. Hence the entropies of these states concur.

The $q$-average of $q$-entropies of particular outputs $\boldsymbol{\rho}_{j}^{\prime}$ is bounded from above by the input $q$-entropy. The relation (17) can be treated as an extension of the second bound in theorem 1 of Ref. [31]. That bound on the average output entropy is yielded from Eq. (17) by taking $q=1$. Incidentally, the first inequality of theorem 1 in Ref. [31] states that the Holevo quantity is not larger than the exchange entropy. The strong subadditivity of the von Neumann entropy plays a principal role in its proof. In classical regime, the $q$-entropy does enjoy the strong subadditivity for $q>1$ [34], however this is an open question for quantum $q$-entropies.

We now illustrate the above result with the depolarizing channel. This well-known model represents a decohering qubit [35]. In the example, we will denote the identity $2 \times 2$-matrix by $\mathbb{1}$ and the usual Pauli matrices by $\boldsymbol{\sigma}_{x}, \boldsymbol{\sigma}_{y}$, and $\boldsymbol{\sigma}_{z}$, respectively. The Kraus operators can be written as [35]

$$
\mathrm{K}_{0}=\sqrt{1-p} \mathbb{1}, \quad \mathrm{K}_{j}=\sqrt{\frac{p}{3}} \boldsymbol{\sigma}_{j}
$$

where $j=x, y, z$. Here, the parameter $p \in[0 ; 1]$ characterizes a probability that an error occurs. Since the case $p=0$ gives the identity map, we will further take $p \neq 0$. In terms of the Bloch vector $\vec{s}=\left(s_{x}, s_{y}, s_{z}\right)$, the input density matrix is written as

$$
\rho=\frac{1}{2}(\mathbb{1}+\vec{s} \cdot \vec{\sigma}) .
$$

Its eigenvalues are $(1 \pm|\vec{s}|) / 2$. For impure states, we have $|\vec{s}|<1$. The particular outputs of the channel are the following. First, one gives $\boldsymbol{\rho}_{0}^{\prime}=\boldsymbol{\rho}$. For $j=x, y, z$, the Bloch vector of $\boldsymbol{\rho}_{j}^{\prime}$ has the same $j$-component as the input one, whereas other two components are with reversed sign. The four particular outputs have the same eigenvalues as the input $\boldsymbol{\rho}$, whence $\mathrm{H}_{q}\left(\boldsymbol{\rho}_{j}^{\prime}\right)=\mathrm{H}_{q}(\boldsymbol{\rho})$ for all $j$. Further, the probabilities are $p_{0}=1-p$ and $p_{j}=p / 3$ for $j=x, y, z$. With impure $\boldsymbol{\rho}$, the relation (17) is then reduced to

$$
f_{q}(p):=(1-p)^{q}+3^{1-q} p^{q} \leq 1 .
$$

When $q \geq 1$, the inequality (27) actually holds for all $p \in[0 ; 1]$. Moreover, for $q>1$ and $p \in(0 ; 1]$ the quantity $f_{q}(p)$ is strictly less than 1 . That is, for all impure inputs and non-zero $p$ we obtain

$$
\left\langle\mathrm{H}_{q}\left(\boldsymbol{\rho}_{j}^{\prime}\right)\right\rangle_{q}<\mathrm{H}_{q}(\boldsymbol{\rho}) .
$$

The last follows from the convexity of the function $p \mapsto f_{q}(p), f_{q}(0)=1$ and $f_{q}(1)=3^{1-q}<1$. We have observed a simple test for the validity of Eq. (17). The depolarizing channel gives also an example, in which the relation (17) is obviously violated with $0<q<1$. Here, the function $p \mapsto f_{q}(p)$ is concave, $f_{q}(0)=1$ and $f_{q}(1)=3^{1-q}>1$, whence $f_{q}(p)>1$ for each $p \in(0 ; 1]$. Thus, for all impure inputs and non-zero $p$ we should rewrite the inequality (28) in opposite direction. To this point, we recall that the triangle inequality for the quantum $q$-entropy of degree $q \geq 1$ has been crucial in the proof of Eq. (17).

\section{CONCAVITY PROPERTIES OF THE $(q, s)$-ENTROPY EXCHANGE}

In this section we study some properties of the $(q, s)$-entropy exchange, which were introduced in Ref. [15]. To do so, we explicitly separate the principal system $Q$ from the imaginary reference system $R$ and environment $E$. The 
Hilbert spaces are denoted by $\mathcal{H}_{Q}, \mathcal{H}_{R}$, and $\mathcal{H}_{E}$, respectively. Under the action of quantum channel $\Phi^{Q}$, the input state $\boldsymbol{\rho}^{Q}$ of system $Q$ is transformed into the output state $\Phi^{Q}\left(\boldsymbol{\rho}^{Q}\right)$. To see the entanglement transmission, we consider a purification $\left|\psi^{Q R}\right\rangle \in \mathcal{H}_{Q} \otimes \mathcal{H}_{R}$, which is mapped into the final density matrix

$$
\rho^{Q^{\prime} R^{\prime}}=\Phi^{Q} \otimes \mathrm{id}^{R}\left(\left|\psi^{Q R}\right\rangle\left\langle\psi^{Q R}\right|\right)
$$

The state of the system $R$ itself is not changed, i.e., $\operatorname{Tr}_{Q}\left(\boldsymbol{\rho}^{Q^{\prime} R^{\prime}}\right)=\operatorname{Tr}_{Q}\left(\left|\psi^{Q R}\right\rangle\left\langle\psi^{Q R}\right|\right)$. The entanglement fidelity is defined as [7]

$$
F\left(\boldsymbol{\rho}^{Q}, \Phi^{Q}\right):=\left\langle\psi^{Q R}\left|\boldsymbol{\rho}^{Q^{\prime} R^{\prime}}\right| \psi^{Q R}\right\rangle
$$

The notion of entropy exchange is very important, since it is related to channel capacity. In our notation, the von Neumann entropy exchange is put as

$$
\mathrm{H}_{1}\left(\boldsymbol{\rho}^{Q^{\prime} R^{\prime}}, \Phi^{Q}\right):=-\operatorname{Tr}\left(\boldsymbol{\rho}^{Q^{\prime} R^{\prime}} \ln \boldsymbol{\rho}^{Q^{\prime} R^{\prime}}\right) .
$$

This quantity was independently introduced by Lindblad [16] and Schumacher [7]. Putting an environment E, we have an environmental representation of the quantum channel $\Phi^{Q}$. It is written in terms of unitary operator $\widetilde{U}$ on $\mathcal{H}_{E} \otimes \mathcal{H}_{Q}$ and pure state $\left|e_{0}\right\rangle \in \mathcal{H}_{E}$ as

$$
\Phi^{Q}\left(\boldsymbol{\rho}^{Q}\right)=\operatorname{Tr}_{E}\left\{\widetilde{\mathrm{U}}\left(\left|e_{0}\right\rangle\left\langle e_{0}\right| \otimes \boldsymbol{\rho}^{Q}\right) \widetilde{U}^{\dagger}\right\}
$$

where the partial trace is taken over an environment. As the final state $\left(\widetilde{\mathbf{U}} \otimes \mathbb{1}^{R}\right)\left|e_{0}\right\rangle \otimes\left|\psi^{Q R}\right\rangle$ of the system $E Q R$ is pure, the output matrices $\rho^{E^{\prime}} \in \mathcal{L}_{+}\left(\mathcal{H}_{E}\right)$ and $\rho^{Q^{\prime} R^{\prime}} \in \mathcal{L}_{+}\left(\mathcal{H}_{Q} \otimes \mathcal{H}_{R}\right)$ have the same non-zero eigenvalues. So the right-hand side of Eq. (31) is equal to the von Neumann entropy of the output state $\boldsymbol{\rho}^{E^{\prime}}$. Using the entropic functional (7), we define the $(q, s)$-entropy exchange as [15]

$$
\mathrm{E}_{q}^{(s)}\left(\boldsymbol{\rho}^{Q}, \Phi^{Q}\right):=\mathrm{E}_{q}^{(s)}\left(\boldsymbol{\rho}^{Q^{\prime} R^{\prime}}\right)=\mathrm{E}_{q}^{(s)}\left(\boldsymbol{\rho}^{E^{\prime}}\right)
$$

This functional quantifies an amount of the $(q, s)$-entropy introduced by the channel $\Phi^{Q}$ into an initially pure environment $E$. The final state of $E$ can be expressed as [1]

$$
\boldsymbol{\rho}^{E^{\prime}}=\sum_{i j} w_{i j}\left|e_{i}\right\rangle\left\langle e_{j}\right|
$$

where $w_{i j}=\operatorname{Tr}\left(\mathrm{K}_{i} \boldsymbol{\rho}^{Q} \mathrm{~K}_{j}^{\dagger}\right)$ are entries of the matrix $\mathrm{W}=\left[\left[w_{i j}\right]\right]$. Using this matrix, the $(q, s)$-entropy exchange (33) can be rewritten as

$$
\mathrm{E}_{q}^{(s)}\left(\boldsymbol{\rho}^{Q}, \Phi^{Q}\right)=\frac{1}{(1-q) s}\left\{\left[\operatorname{Tr}\left(\mathrm{W}^{q}\right)\right]^{s}-1\right\} .
$$

In a similar manner [7], the entanglement fidelity is represented by $F\left(\boldsymbol{\rho}^{Q}, \Phi^{Q}\right)=\sum_{j}\left|\operatorname{Tr}\left(\boldsymbol{\rho}^{Q} \mathrm{~K}_{j}\right)\right|^{2}$. Since the main definitions are already given, we now simplify the notation to $\mathrm{E}_{q}^{(s)}(\boldsymbol{\rho}, \Phi)$ and $F(\boldsymbol{\rho}, \Phi)$. We will omit the superscript $Q$ whenever density matrices and quantum operations are related to the principal system $Q$ only. We shall now pose convexity or concavity properties of the $(q, s)$-entropy exchange with respect to its arguments. A short comment should be made here. For any $\mathrm{X} \in \mathcal{L}_{+}(\mathcal{H})$ and non-zero real number $q$, one defines the functional

$$
\mathrm{G}_{q}(\mathrm{X}):=\left[\operatorname{Tr}\left(\mathrm{X}^{q}\right)\right]^{1 / q} .
$$

We claim that this functional satisfies

$$
\begin{array}{lr}
\mathrm{G}_{q}(\mathrm{X}+\mathrm{Z}) \geq \mathrm{G}_{q}(\mathrm{X})+\mathrm{G}_{q}(\mathrm{Z}) & (0<q<1) \\
\mathrm{G}_{q}(\mathrm{X}+\mathrm{Z}) \leq \mathrm{G}_{q}(\mathrm{X})+\mathrm{G}_{q}(\mathrm{Z}) & (1<q<\infty) .
\end{array}
$$

For $q=1$, we have $\mathrm{G}_{q}(\mathrm{X}+\mathrm{Z})=\mathrm{G}_{q}(\mathrm{X})+\mathrm{G}_{q}(\mathrm{Z})$. The relations (37) and (38) can be considered as a tracial version of the classical Minkowski inequality. Since operators $X$ and $Z$ do not generally commute, the Minkowski inequality for tuples of numbers is not sufficient for justifying Eqs. (37) and (38). The formula (38) is actually no more than the triangle inequality for the Schatten $q$-norm (see, e.g., sect. 7.1 of Ref. [36]). Assuming $q \in(0 ; 1)$, the inequality (37) can be regarded as the super-additivity inequality for the Schatten $q$-anti-norm. Anti-norms of positive matrices are extensively treated in the paper [37]. In principle, the relation (37) can be obtained as a special case of proposition 3.7 of Ref. [37]. In Appendix [A, we give another proof, which is closely related to the classical Minkowski inequality for number tuples. Using Eqs. (37) and (38), the authors of Ref. [5] have examined concavity properties of the quantum $(q, s)$-entropy. Note that the Minkowski inequality was used in Ref. [5] with no comments on the non-commutativity. It is for this reason that we prove Eq. (37) in Appendix A. 
Proposition 2 Let $\Phi, \Psi$ be quantum channels, and let $\boldsymbol{\rho}, \varrho \in \mathcal{L}_{+}(\mathcal{H})$ be density matrices. In the parameter range

$$
\left\{(q, s): 0<q \leq 1,-\infty<s \leq q^{-1}\right\} \bigcup\left\{(q, s): 1 \leq q, q^{-1} \leq s<+\infty\right\},
$$

for each $\theta \in[0 ; 1]$, the $(q, s)$-entropy exchange satisfies

$$
\begin{aligned}
& \mathrm{E}_{q}^{(s)}(\theta \boldsymbol{\rho}+(1-\theta) \boldsymbol{\varrho}, \Phi) \geq \theta \mathrm{E}_{q}^{(s)}(\boldsymbol{\rho}, \Phi)+(1-\theta) \mathrm{E}_{q}^{(s)}(\varrho, \Phi) \\
& \mathrm{E}_{q}^{(s)}(\boldsymbol{\rho}, \theta \Phi+(1-\theta) \Psi) \geq \theta \mathrm{E}_{q}^{(s)}(\boldsymbol{\rho}, \Phi)+(1-\theta) \mathrm{E}_{q}^{(s)}(\boldsymbol{\rho}, \Psi) .
\end{aligned}
$$

Proof. Summarizing the results of the works [ $[,[6]$, we state the following. In the parameter domain (39), for each $\theta \in[0 ; 1]$ there holds

$$
\mathrm{E}_{q}^{(s)}(\theta \boldsymbol{\rho}+(1-\theta) \varrho) \geq \theta \mathrm{E}_{q}^{(s)}(\boldsymbol{\rho})+(1-\theta) \mathrm{E}_{q}^{(s)}(\varrho) .
$$

Using Eqs. (37) and (38), the writers of Ref. [5] have proved this inequality in the domain (39) for any $s \neq 0$. The concavity for $0<q \leq 1$ and $s=0$, i.e., for Renyi's $q$-entropies of order $q \in(0 ; 1]$, was shown in theorem 2 of Ref. [6].

Consider the inputs $\rho$ and $\varrho$. An action of the channel $\Phi$ results in the matrices $\mathrm{W}_{\boldsymbol{\rho}}$ and $\mathrm{W}_{\varrho}$ with elements $\operatorname{Tr}\left(\mathrm{K}_{i} \boldsymbol{\rho} \mathrm{K}_{j}^{\dagger}\right)$ and $\operatorname{Tr}\left(\mathrm{K}_{i} \varrho \mathrm{K}_{j}^{\dagger}\right)$, respectively. For the input $\theta \boldsymbol{\rho}+(1-\theta) \varrho$, the corresponding matrix is written as

$$
\mathrm{W}=\theta \mathrm{W}_{\rho}+(1-\theta) \mathrm{W}_{\varrho} .
$$

Combining this with Eq. (35) and the property (42), we obtain the first claim (40). To prove Eq. (41), we recall the meaning of the systems $Q$ and $R$ from the definitions (29)-(31). By $\rho_{\Phi}^{Q^{\prime} R^{\prime}}$ and $\boldsymbol{\rho}_{\Psi}^{Q^{\prime}} R^{\prime}$, we respectively denote density operators generated by channels $\Phi$ and $\Psi$ in the sense of Eq. (29). By linearity, one further obtains

$$
(\theta \Phi+(1-\theta) \Psi) \otimes \operatorname{id}^{R}\left(\left|\psi^{Q R}\right\rangle\left\langle\psi^{Q R}\right|\right)=\theta \boldsymbol{\rho}_{\Phi}^{Q^{\prime} R^{\prime}}+(1-\theta) \boldsymbol{\rho}_{\Psi}^{Q^{\prime} R^{\prime}} .
$$

Combining this with Eq. (33) and the property (42) finally leads to the second claim (41).

Proposition 2 states that the $(q, s)$-entropy exchange is concave in its first entry as well as in its second entry. For the case $s=1$, i.e., for the Tsallis entropy exchange, these facts were noted in Ref. [32]. Fano type inequalities have also been obtained in that paper. Bounds of such a kind can also be derived for the $(q, s)$-entropy exchange.

\section{FANO TYPE INEQUALITIES FOR THE $(q, s)$-ENTROPY EXCHANGE}

In this section, we use the results of Ref. [32] to obtain upper estimates of the Fano type for the $(q, s)$-entropy exchange with arbitrary values of the parameters. The following statement takes place.

Proposition 3 For $q>0$ and all real $s$, the $(q, s)$-entropy exchange is bounded from above as

$$
\mathrm{E}_{q}^{(s)}(\boldsymbol{\rho}, \Phi) \leq \frac{1}{1-q} \ln _{1-s}\left\{1+(1-q) \widetilde{H}_{q}(F(\boldsymbol{\rho}, \Phi))+(1-q)[1-F(\boldsymbol{\rho}, \Phi)]^{q} \ln _{q}\left(d^{2}-1\right)\right\} .
$$

Proof. It has been shown in Ref. [32] that for all $q>0$, there holds

$$
\mathrm{H}_{q}(\boldsymbol{\rho}, \Phi) \leq \widetilde{H}_{q}(F(\boldsymbol{\rho}, \Phi))+[1-F(\boldsymbol{\rho}, \Phi)]^{q} \ln _{q}\left(d^{2}-1\right) .
$$

In the limit $q=1$, this bound reduces to the standard quantum Fano inequality. So we further assume $q \neq 1$. Suppose also that $s \neq 0$. According to the definitions (5) and (7), we can write

$$
\mathrm{E}_{q}^{(s)}(\boldsymbol{\rho}, \Phi)=\frac{1}{(1-q) s}\left\{\left[1+(1-q) \mathrm{H}_{q}(\boldsymbol{\rho}, \Phi)\right]^{s}-1\right\} .
$$

The function $x \mapsto(1-q)^{-1} s^{-1}\left\{(1+(1-q) x)^{s}-1\right\}$ is increasing for those values of $x$ that obey $1+(1-q) x>0$. Indeed, its derivative is then positive. Combining this with Eqs. (46) and (47) gives the relation (46) with $s \neq 0$, since we have a functional identity

$$
\frac{y^{s}-1}{(1-q) s}=\frac{1}{1-q} \ln _{1-s}(y)
$$


In line with the definitions (5) and (8), we further write

$$
\mathrm{R}_{q}(\boldsymbol{\rho}, \Phi)=\frac{1}{1-q} \ln \left\{1+(1-q) \mathrm{H}_{q}(\boldsymbol{\rho}, \Phi)\right\} .
$$

We now observe that the function $x \mapsto(1-q)^{-1} \ln (1+(1-q) x)$ monotonically increases with values of $x$ such that $1+(1-q) x>0$. Combining this with Eqs. (46) and (49) leads to the relation (46) with $s=0$.

For many values of the parameters, the above upper bounds can slightly be simplified. In the parameter range

$$
\{(q, s): 0<q \leq 1,-\infty<s \leq 1\} \bigcup\{(q, s): 1 \leq q, 1 \leq s<+\infty\}
$$

there holds Eq. (46) with $\mathrm{E}_{q}^{(s)}(\boldsymbol{\rho}, \Phi)$ instead of $\mathrm{H}_{q}(\boldsymbol{\rho}, \Phi)$, namely

$$
\mathrm{E}_{q}^{(s)}(\boldsymbol{\rho}, \Phi) \leq \widetilde{H}_{q}(F(\boldsymbol{\rho}, \Phi))+[1-F(\boldsymbol{\rho}, \Phi)]^{q} \ln _{q}\left(d^{2}-1\right) .
$$

The case $s=1$ is merely the bound (46) itself. So we will prove Eq. (51) for $s \neq 1$ (and also $q \neq 1$ ). Assume that $s \neq 0$ as well. For positive $x$, we can write the inequality

$$
\frac{(1+(1-q) x)^{s}-1}{(1-q) s}=\int_{0}^{x}(1+(1-q) t)^{s-1} d t \leq \int_{0}^{x} d t=x
$$

whenever $(1+(1-q) t)^{s-1} \leq 1$. The latter holds in the following two cases: (i) $q<1$ and $s<1$; (ii) $1<q$ and $1<s$. Except for the values $q=1$ and $s=0,1$, these cases fully cover the parameter range (50). Combining this with Eqs. (46) and (47) completes the proof of (51) for $s \neq 0,1$. The case $s=0$, when Rényi's entropy is dealt with, follows from Eq. (49) and the inequality

$$
\frac{1}{1-q} \ln (1+(1-q) x)=\int_{0}^{x} \frac{d t}{1+(1-q) t} \leq \int_{0}^{x} d t=x
$$

which holds for $q<1$. So we obtain Eq. (51) for $0<q<1$ and $s=0$.

The formulas (45) and (51) provide upper estimates of the Fano type on the $(q, s)$-entropy exchange. These estimates are expressed in terms of the entanglement fidelity and the corresponding binary $q$-entropy. The following treatment can be given here. The first summand in the right-hand side of Eq. (51), i.e., the corresponding binary $q$-entropy, is small when the entanglement fidelity is close to either zero or one. But only for the former the second summand in the right-hand side of Eq. (51) becomes almost maximal. When the $(q, s)$-entropy exchange is large, the entanglement fidelity should be small enough. That is, the entanglement between $Q$ and $R$ has not been well preserved. On the other hand, if the channel $\Phi$ does preserve the entanglement appropriately, then its $(q, s)$-entropy exchange is small. The $(q, s)$-entropy exchange succeeds to the standard entropy exchange.

\section{UPPER BOUNDS ON THE MAP $(q, s)$-ENTROPY}

In this section, we will further examine the map $(q, s)$-entropies introduced in Ref. [15]. For quantum channel $\Phi$ with the rescaled dynamical matrix $\boldsymbol{\sigma}(\Phi)$, we define the map $(q, s)$-entropy by

$$
\mathrm{M}_{q}^{(s)}(\Phi):=\mathrm{E}_{q}^{(s)}(\boldsymbol{\sigma}(\Phi))
$$

This is an extension of the standard map entropy introduced in Ref. 8] as the von Neumann entropy of $\boldsymbol{\sigma}(\Phi)$. Essential properties of the standard map entropy were examined in Ref. [12]. The map entropy is used to characterize the decoherent behavior of given quantum channel. If the input of the channel is taken to be completely mixed, then the entropy exchange coincides with the map entropy [31]. The same can be shown for the $(q, s)$-entropy exchange and the map $(q, s)$-entropy. Few additivity properties of the map entropy (54) have been analyzed in Ref. [15]. These results were based on some extension of the Lindblad inequality [16]. Recall that the entropy exchange is bounded from above by the sum of the input and output von Neumann entropies of the principal quantum system. Hence, estimates on the output entropy of completely mixed input state can be obtained. Such a question has arisen within studies of so-called "additivity conjecture" concerning a product quantum channel (for details, see the paper [12] and references therein). A relevant extension of the Lindblad inequality to the $(q, s)$-entropy entropy exchange is written as follows. For $q>1$ and $s \geq q^{-1}$, we have [15]

$$
\left|\mathrm{E}_{q}^{(s)}(\Phi(\boldsymbol{\rho}))-\mathrm{E}_{q}^{(s)}(\boldsymbol{\rho})\right| \leq \mathrm{E}_{q}^{(s)}(\boldsymbol{\rho}, \Phi) \leq \mathrm{E}_{q}^{(s)}(\Phi(\boldsymbol{\rho}))+\mathrm{E}_{q}^{(s)}(\boldsymbol{\rho}),
$$


including permutations of the three entropies. The proof of Eq. (55) is based on the subadditivity of the quantum $(q, s)$-entropy for $q>1$ and $s \geq q^{-1}[6]$. The latter has been obtained by a relevant extension of the reasons given for quantum Tsallis' entropy in Ref. [33]. Taking the completely mixed state $\boldsymbol{\rho}_{*}=\mathbb{1} / d$ as an input, the formula (55) leads to an upper bound on the map $(q, s)$-entropy, namely

$$
\mathrm{M}_{q}^{(s)}(\Phi) \leq \mathrm{E}_{q}^{(s)}\left(\Phi\left(\boldsymbol{\rho}_{*}\right)\right)+\frac{1}{s} \ln _{q}\left(d^{s}\right)
$$

This bound is expressed in terms of the output entropy and the dimensionality. It turns out that a stronger bound can be derived. Moreover, it is applicable for arbitrary values of the parameters. We have the following statement.

Proposition 4 Let $\Phi$ be a quantum channel, and let $\boldsymbol{\rho}_{*}$ be the completely mixed state on d-dimensional space $\mathcal{H}$. For $q>0$ and all real $s$, the map $(q, s)$-entropy is bounded from above as

$$
\begin{aligned}
& \mathrm{M}_{q}^{(s)}(\Phi) \leq d^{(1-q) s} \mathrm{E}_{q}^{(s)}\left(\Phi\left(\boldsymbol{\rho}_{*}\right)\right)+\frac{1}{s} \ln _{q}\left(d^{s}\right) \quad(s \neq 0) \\
& \mathrm{M}_{q}^{(0)}(\Phi) \leq \mathrm{R}_{q}\left(\Phi\left(\boldsymbol{\rho}_{*}\right)\right)+\ln d .
\end{aligned}
$$

Proof. In Ref. 38], we have derived inequalities between the unified entropies of a composite quantum system and one of its subsystems. Namely, for the final density matrix (29) and all $q>0 \neq 1$ there holds

$$
\begin{aligned}
& \mathrm{E}_{q}^{(s)}\left(\boldsymbol{\rho}^{Q^{\prime} R^{\prime}}\right) \leq d^{(1-q) s} \mathrm{E}_{q}^{(s)}\left(\Phi^{Q}\left(\boldsymbol{\rho}^{Q}\right)\right)+\frac{1}{s} \ln _{q}\left(d^{s}\right) \quad(s \neq 0), \\
& \mathrm{R}_{q}\left(\boldsymbol{\rho}^{Q^{\prime} R^{\prime}}\right) \leq \mathrm{R}_{q}\left(\Phi^{Q}\left(\boldsymbol{\rho}^{Q}\right)\right)+\ln d .
\end{aligned}
$$

Here, we use the notation with superscripts of the systems $Q$ and $R$. The inequalities (59) and (60) follow from relations, which describe a change of some symmetric norms and anti-norms under the operation of partial trace [38]. Taking the completely mixed state as the channel input, the left-hand sides of Eqs. (59) and (60) are equal to the corresponding map entropies. Omitting the superscripts, we have arrived at the claims (57) and (58).

The inequalities (57) and (58) give upper bounds on the map $(q, s)$-entropies for all considered values of the parameters $q$ and $s$. The formula (56) provides an upper bound only for $q>1$ and $s \geq q^{-1}$. In this case, the power exponential $(1-q) s$ in the right-hand side of Eq. (57) is negative. The new bound (57) is stronger than Eq. (56). In the standard limit $q \rightarrow 1$, these bounds obviously coincide. Similar bounds in terms of the output ( $q, s)$-entropies have been presented in Ref. [38]. Replacing the dimensionality with the rank of the dynamical matrix and the $\boldsymbol{\rho}_{*}$ with arbitrary input $\boldsymbol{\rho}$, the right-hand sides of (57) and (58) provide upper bounds on the corresponding input entropies. All the bounds follow from inequalities relating certain norms and anti-norms before and after partial trace 38. We may use the results (57) and (58) for estimating the map $(q, s)$-entropies from above, when the corresponding output entropies are known, exactly or approximately.

\section{CONCLUSION}

In this paper, we have discussed some channel characteristics expressed in terms of generalized quantum entropies. Generally, discussed properties are dependent on the parameter values. In many respects, generalized-entropy characteristics can be treated similarly to the ones based on the von Neumann entropy. It turned out that for any quantum channel and $q \geq 1$, the $q$-average of $q$-entropies of particular outputs is bounded from above by the corresponding input entropy. This result is an extension of the bound given for the von Neumann output and input entropies in Ref. [31]. In a wide range of parametric values, the $(q, s)$-entropy exchange enjoys concavity in any of its two entries. Using Fano type quantum inequality of Ref. [32], similar estimates on the $(q, s)$-entropy exchange have been derived for all acceptable values of the parameters. We also obtain some upper bounds on the map $(q, s)$-entropy introduced in Ref. [15]. Thus, the presented results can be regarded as a supplement and development of the previously given facts on entropic characteristics of quantum channels.

\section{Acknowledgments}

The author is grateful to Karol Życzkowski for helpful correspondence. 


\section{Appendix A: A tracial inequality of Minkowski type}

In this section, we will prove Eq. (37) on base of the classical Minkowski inequality. For strictly positive operators, we can also allow negative exponential $q<0$. Let $x$ be a $d$-tuple of strictly positive numbers. For $q \neq 0$, we introduce the function

$$
G_{q}(x):=\left(\sum_{j=1}^{d} x_{j}^{q}\right)^{1 / q}
$$

We can write $\mathrm{G}_{q}(\mathrm{X})=G_{q}(\lambda(\mathrm{X}))$, where $\lambda(\mathrm{X})$ denotes a $d$-tuple of positive eigenvalues of $\mathrm{X}$. For $q \geq 1$, the right-hand side of Eq. (A1) gives the symmetric gauge function assigned to the Schatten $q$-norm. The function (A1) clearly obeys permutation symmetry and homogeneity, i.e., $G_{q}(\alpha x)=\alpha G_{q}(x)$ with factor $\alpha>0$. In line with the usual Minkowski inequality (see, e.g., theorem 25 in Ref. [39]), for $q<1 \neq 0$ there holds

$$
G_{q}(x+y+\ldots+z) \geq G_{q}(x)+G_{q}(y)+\ldots+G_{q}(z) .
$$

The cases of equality in Eq. (A2) and more details are provided in Ref. [39]. The following statement takes place.

Proposition 5 Let $\mathcal{H}$ be finite-dimensional Hilbert space and $\mathrm{X}, \mathrm{Z} \in \mathcal{L}_{++}(\mathcal{H})$. For all non-zero $q<1$, the functional (36) satisfies

$$
\mathrm{G}_{q}(\mathrm{X}+\mathrm{Z}) \geq \mathrm{G}_{q}(\mathrm{X})+\mathrm{G}_{q}(\mathrm{Z})
$$

Proof. Due to the Ky Fan maximum principle [40], the vector $\lambda(\mathbf{X}+\mathbf{Z})$ is majorized by $\lambda(\mathbf{X})+\lambda(\mathbf{Z})$. Namely, for all $k=1, \ldots, d$ there holds

$$
\sum_{j=1}^{k} \lambda_{j}(\mathrm{X}+\mathrm{Z})^{\downarrow} \leq \sum_{j=1}^{k} \lambda_{j}(\mathrm{X})^{\downarrow}+\sum_{j=1}^{k} \lambda_{j}(\mathrm{Z})^{\downarrow}
$$

with the equality $\operatorname{Tr}(\mathbf{X}+\mathbf{Z})=\operatorname{Tr}(\mathbf{X})+\operatorname{Tr}(\mathbf{Z})$. Here the arrows down imply that the eigenvalues should be put in the decreasing order. It is well known that there exists a doubly stochastic matrix $\mathrm{S}$ such that

$$
\lambda(\mathbf{X}+\mathbf{Z})^{\downarrow}=\mathrm{S}\left[\lambda(\mathbf{X})^{\downarrow}+\lambda(\mathbf{Z})^{\downarrow}\right]
$$

For details, see point 4.3 .33 of Ref. [41]. The Birkhoff theorem says that each doubly stochastic matrix is a convex combination of finitely many permutation matrices [41], that is

$$
\mathrm{S}=\sum_{i} \alpha_{i} \mathrm{P}_{i}, \quad \alpha_{i} \geq 0, \quad \sum_{i} \alpha_{i}=1
$$

Combining this fact with the inequality (A2) and using both the homogeneity and symmetry, we then obtain

$$
\begin{aligned}
& G_{q}\left(\lambda(\mathrm{X}+\mathrm{Z})^{\downarrow}\right)=G_{q}\left(\sum_{i} \alpha_{i} \mathrm{P}_{i}\left[\lambda(\mathrm{X})^{\downarrow}+\lambda(\mathrm{Z})^{\downarrow}\right]\right) \\
& \geq \sum_{i} \alpha_{i} G_{q}\left(\mathrm{P}_{i} \lambda(\mathrm{X})^{\downarrow}\right)+\sum_{i} \alpha_{i} G_{q}\left(\mathrm{P}_{i} \lambda(\mathrm{Z})^{\downarrow}\right) \\
& =\sum_{i} \alpha_{i} G_{q}\left(\lambda(\mathrm{X})^{\downarrow}\right)+\sum_{i} \alpha_{i} G_{q}\left(\lambda(\mathrm{Z})^{\downarrow}\right) .
\end{aligned}
$$

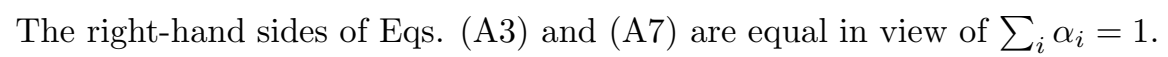

[1] M. A. Nielsen, I. L. Chuang, Quantum Computation and Quantum Information (Cambridge University Press, Cambridge, 2000)

[2] I. Bengtsson, K. Życzkowski, Geometry of Quantum States: An Introduction to Quantum Entanglement (Cambridge University Press, Cambridge, 2006)

[3] A. Rényi, In: J. Neyman (Ed.), Proceedings of 4th Berkeley Symposium on Mathematical Statistics and Probability, vol. I (University of California Press, Berkeley, 1961) 547

[4] C. Tsallis, J. Stat. Phys. 52, 479 (1988)

[5] X. Hu, Z. Ye, J. Math. Phys. 47, 023502 (2006)

[6] A. E. Rastegin, J. Stat. Phys. 143, 1120 (2011) 
[7] B. Schumacher, Phys. Rev. A 54, 2614 (1996)

[8] K. Życzkowski, I. Bengtsson, Open Sys. Inf. Dyn. 11, 3 (2004)

[9] W. Roga, Z. Puchała, Ł. Rudnicki, K. Życzkowski, arXiv:1206.2536 [quant-ph]

[10] S. Wehner, A. Winter, New J. Phys. 12, 025009 (2010)

[11] I. Bialynicki-Birula, Ł. Rudnicki, Entropic Uncertainty Relations in Quantum Physics, In: K. D. Sen (Ed.), Statistical Complexity, 1 (Springer, Berlin, 2011) DOI: 10.1007/978-90-481-3890-6-1

[12] W. Roga, M. Fannes, K. Życzkowski, Int. J. Quantum Inf. 9, 1031 (2011)

[13] P. W. Shor, Commun. Math. Phys. 246, 453 (2004)

[14] F. G. S. L. Brandão, M. Horodecki, Open Sys. Inf. Dyn. 17, 31 (2010)

[15] A. E. Rastegin, J. Phys. A: Math. Theor. 45, 045302 (2012)

[16] G. Lindblad, In: C. Bendjaballah, O. Hirota, S. Reynaud (Eds.), Quantum Aspects of Optical Communications, Lecture Notes in Physics, vol. 378 (Springer, Berlin, 1991) 71

[17] J. Havrda, F. Charvát, Kybernetika 3, 30 (1967)

[18] E. M. F. Curado, C. Tsallis, J. Phys. A: Math. Gen. 24, L69 (1991)

[19] V. Majerník, E. Majerníková, S. Shpyrko, Cent. Eur. J. Phys. 3, 393 (2003)

[20] S. Wehner, A. Winter, J. Math. Phys. 49, 062105 (2008)

[21] A. E. Rastegin, J. Phys. A: Math. Theor. 43, 155302 (2010)

[22] A. E. Rastegin, J. Phys. A: Math. Theor. 44, 095303 (2011)

[23] A. E. Rastegin, Phys. Scr. 84, 057001 (2011)

[24] A. E. Rastegin, Int. J. Theor. Phys. 51, 1300 (2012)

[25] G. Wilk, Z. Włodarczyk, Cent. Eur. J. Phys. 10, 568 (2012)

[26] G. A. Raggio, J. Math. Phys. 36, 4785 (1995)

[27] J. Watrous, Theory of Quantum Information (Lecture notes for CS 798, University of Waterloo, 2008) http://www.cs.uwaterloo.ca/ watrous/quant-info/lecture-notes/

[28] A. Jamiołkowski, Rep. Math. Phys. 3, 275 (1972)

[29] M.-D. Choi, Linear Algebra Appl. 10, 285 (1975)

[30] J. A. Miszczak, Int. J. Mod. Phys. C 22, 897 (2011)

[31] W. Roga, M. Fannes, K. Życzkowski, Phys. Rev. Lett. 105, 040505 (2011)

[32] A. E. Rastegin, Quantum Inf. Process. 11, 1895 (2012)

[33] K. Audenaert, J. Math. Phys. 48, 083507 (2007)

[34] S. Furuichi, J. Math. Phys. 47, 023302 (2006)

[35] J. Preskill, Quantum Computation and Information (Lecture notes for Physics 229, California Institute of Technology, 1998) http://www.theory.caltech.edu/people/preskill/ph229/

[36] E. A. Carlen, Trace Inequalities and Quantum Entropy: An Introductory Course (Lecture course given at "Entropy and the Quantum", Tucson, Arizona, 2009) http://www.mathphys.org/AZschool/material/AZ09-carlen.pdf

[37] J.-C. Bourin, F. Hiai, Intern. J. Math. 22, 1121 (2011)

[38] A. E. Rastegin, J. Stat. Phys. 148, 1040 (2012)

[39] G. H. Hardy, J. E. Littlewood, G. Polya, Inequalities (Cambridge University Press, London, 1934)

[40] K. Fan, Proc. Nat. Acad. Sci. USA 35, 652 (1949)

[41] R. A. Horn, C. R. Johnson, Matrix Analysis (Cambridge University Press, Cambridge, 1985) 